from the presence of a new growth it is of interest to note that the passage of an opaque meal given by the mouth may be quite unobstructed. In other words, there may be no delay whatever and yet the obstruction will be complete to the opaque enema. Various suggestions have been made to account for this phenomenon, but my impression is that a channel through the new growth is moulded-probably into the shape of a funnel-by the onward passage of faeces, a valve-like closure taking place when the enema is administered. In any case, it is rare for a colonic growth to be present without giving rise to definite obstruction to the passage of the opaque enema.

The first indication of this obstruction is the sudden increase in colonic tension-imparted to those who are able to appreciate it-through the rubber ball of the modified Higginson syringe already mentioned. The column of the barium fluid ends abruptly as it comes up against the growth. In other words, it is straight-faced. Gradually a small amount may be enticed through the stricture. Care must be taken at this stage not to use force, otherwise damage may be done or the enema may be returned. Once the emulsion has got through the narrowed section, the extent of the disease can be gauged. The narrowed channel is usually irregular and tortuous, similar to a new oesophageal growth. I smooth in outline, care should be taken to eliminate local spasm; at the same time it must be remembered that in some cases of annular growths the stricture may be smooth, but then it is permanent. Palpation here may be of considerable use, for although no actual tumour can be felt, the rolling of the suspected area of colon from side to side under the fingers will impart a definite sensation of thickening of the wall.

The most difficult section to examine is the pelvic colon and first part of the sigmoid. The coils tend to overlap one another and considerable ingenuity is necessary to disentangle the shadows, especially as palpation is not possible in this portion of the gut. How, by injecting water, this difficulty may be overcome has already been described. A new growth involving the caecum may be orerlooked or a wrong diagnosis of diseased colon may be made, unless care is taken that this portion is fully filled. There is a tendency to what $I$ take to be an air-lock in this region, giving an irregular outline to the caecum. Palpation, however, will help it to fill to its fullest extent, but if this is unsuccessful the object will be achieved by turning the patient on to the face for a few minutes. Dilatation of the distal side of the obstruction is by no means unusual.

As previously mentioned, the opaque enema is the only satisfactory method of detecting the presence of narrowing or distortion of the gut, and the extent of the disease.

\section{Tuberculous Disease.}

Although a rare condition, tuberculous disease may be considered here owing to the fact that it closely resembles a new growth in its radiological appearances.

While tuberculous disease certainly causes narrowing and distortion of the gut, this is usually limited to one side, not involving, as with a new growth, the whole lumen. The proximal half of the colon is commonly implicated. The lesion is usually more extensive and the obstruction is seldom so complete as when caused by a new growth. The history of the case will also assist a correct interpretation of the findings. It is, however, by no means easy to distinguish between these two lesions. Differential diagnosis is also difficult between $(a)$ adhesions, $(b)$ actinomycosis, (c) abscess, and $(d)$ extrinsic tumours.

\section{Colonic Diverticulation.}

This condition is more frequent than is generally supposed, but it does not necessarily give rise to symptoms unless inflammation supervenes. It is by no means uncommon in patients over 45. This pouching or sacculation of the coloil is probably the result of some defect in the muscular coat, congenital or acquired. The diagnosis depends on the filling of these diverticula with the opaque fluid, and are either seen as bud-like projections, while the gut is fully distended, or as button-like shadows left behind after evacuation. They may be sessile or pedunculated, and are situated outside the limit of the gut. Examination immediately after evacuation may show a few of these saccules, but many more will be detected if the examination is made again in twenty-four hours.

I have found that diverticula may remain filled with the opaque fluid for several weeks after the enema, and this even after vigorous irrigation of the colon. What part diverticula play in intestinal toxaemia has yet to be established, but the fact that they may not be emptied even by repeated washings out of the gut at once raises the question whether some of the cases of chronic infection may not be the result of absorption from these diverticula, adding yet another source of infection to the already long list. Diverticula, in fact, may be looked upon as miniature appendices that may become chronically and occasionally acutely infected. A term is wanted to describe the condition when diverticula are present without inflammatory changes. The term "diverticulitis" implies inflammation. I suggest the ter'm " colonic diverticulation."

In all suspected cases, or where the examination has revealed nothing abnormal, an examination after twentyfour hours is essential. Diverticula usually occur in the descending portion or sigmoid, although they may be found in any section of the colon and occasionally along its whole length. If there has been any inflammatory condition present (diverticulitis) it is rare for the opaque fluid to get into the particular direrticulum involved, as the opening into the gut is usually occluded. Other patent diverticula are, however, as a rule present, indicating the nature of the case. Residue in the colonic haustra should not be mistaken for diverticula.

\section{Benign Tumours.}

These are rare and the radiological differentiation almost impossible. Smooth defects in the outline of the lumen, not characteristic of a malignant tumour, may lead one to suspect the simple nature of the lesion. Polypi will cause a smooth defect if large enough, with little or no obstruction, the shadows cast by the gut in the region being rather mottled in appearance. Multiple adenomata give a similar picture.

\section{Chronic Ulcerative Colitis.}

The element of spasm is the main feature here. This leads to a spastic condition of the colon, usually of the descending portion, giving rise to an extensire narrowing of a considerable part of the gut. In rare cases the opaque fluid can be seen adhering to the ulcer surfaces themselves after evacuation. The presence of diverticula may cause symptoms of colitis, and care should be taken not to overlook this condition.

\section{Conclusion.}

While examination by the opaque enema has been $\mathrm{cm}-$ ployed for some years, I feel that a more extended use of this method could be made with advantage in this country. But it must be realized that the examination is beset with pitfalls for the unwary.

If careful attention be given to the technical details the method will prove to be rapid and accurate, and to cause minimum discomfort to the patient.

\section{CONCEALED ACCIDENTAL HAEMORRHAGE} BY

\section{T. H. RICHMOND, O.B.E., M.B., Сн.B., F.R.C.S.F., GYNAECOLOGIST, LONGTON HOSPITAL, STOKE-ON-TRENT.}

Ix addition to the relative rarity of concealed accidental haemorrhage there are several points in the following case of more than passing interest.

On January 1st, 1924, I was asked by her family doctor to see a well developed woman of 26 , who was in the thirty-fifth week of her finst pregiancy. Down to eighteen hours before I saw her she had enjoyed good health and the urine had been free from albimin. enjoyed good health and the urine had been free from albimin. she was seized with severe cramp-like pain in the epigastrium. An hour later she became faint and complained of a sensation of great fullness in the abdomen. She was carried to bed, and shortly afterwards felt sick and vomited without gaining any relief. Down to the time when I saw her every attempt at ingestion of food or to raise her head resulted in a recurrence of the faintness and romiting. The bowels had not acted and she vas unable to say if she had passed flatus. There was increined vas unable to say if she had passed flatus. There was increited
frequency of micturition. While the pain had continued without 
intermission from the onset of the attack, she did not think it had differed in character after the end of the first hour. At no time had she any feeling of air hunger.

She lay in a dazed, apathetic condition, and was markedly pale but of a fawny white rather than the ashy grey colour of secondary anaemia. The pulse was 108 and the temperature $98^{\circ} \mathrm{F}$. The abdomen was greatly distended and gave a sensation of hard tenseness. This distension was chiefly localized in the upper segment and passed under the costal margins. No sounds could be heard on auscultation, nor could any movements be recognized on inspection or palpation. The tenderness she com plained of could not be described as severe. No blood was found in the vagina, nor was there any staining of her clothing. The os was dilated ( $2 \mathrm{~cm}$.), with distended membranes bulging through. Concealed accidental hacmorrhage was diagnosed and Caesarean section recommended. This her husband agreed to, but requested that the uterus should be preserved if found possible. She was removed to Longton Hospital.

At 7.30 p.m. on January 1st, 1924, under a general anaesthetic, the abdomen was opened through a mid-line incision extending from 1 inch above the umbilicus to 1 inch above the pubes. little free sanguineous fluid escaped from the peritoneal cavity On attempting to centralize the uterus it was found impossible to insinuate the hand between it and the costal margins. The distension of the uterus was definitely limited to the body, which was of a slaty grey colour with purplish mottling, while the lower uterine segment, below the anterior peritoneal reflection which was raised to the level of the umbilicus, was elongated and showed neither grey coloration nor mottling. A longitudinal incision through the loose peritoneum over this segment exposed suffcient of it to permit a 5 -inch incision into it without reflecting the bladder downwards. This incision was made in the long axis. No difficulty was experienced in extracting a well developed dead child of about $7 \mathrm{lb}$. by the presenting breech. The skin of the scrotum had desquamated, otherwise the child was in a good state of preservation. The placenta was free in the uterine carity and was removed with three or four laree in the uterine These clots collectively would have filled a half-pint measure. Simultaneous with the extraction of the child 1 c.cm. of pituitar extract was given subcutaneously and the uterus lifted out of the abdominal cavity. It remained flabby and toncless, and except while directly compressed there was a steady oozing from its cavity. After fifteen minutes' compression without satisfactory result gr. $1 / 100$ ergotinine citrate was injected directly into the uterine muscle. This had the desired effect, and the uterus was preserved. The placenta was of normal shape and size, but four-fifths of the The placenta was of normal shape and size, but four-fifths of the
villi showed that they had been compressed. The remainder occupied an arc embracing a third of the periphery of the placenta. When the uterus was removed from the abdomina cavity, within a minute of giving pituitary extract, the iniestines were seen collapsed and ivory white in colour: this persisted during the lime the abdomen was open. The incisions in the lower uterine segment and in the peritoneum over it were closed separately with continuous catgut and the abdomen closed in layers.

Except for severe epigastric pain which lasted for two days convalescence was uneventful, and she went home on January 20th; the uterus had then contracted down to the size of a two to three months' pregnancy.

While concealed accidental haemorrhage is one of the rarest complications of pregnancy ( 1 in 15,000 or more cases), it is one of the most serious. In all but those in which the haemorrhage has been trivial the child nust die in utero, and in those severer cases the uterine muscle is so damaged and its power of contraction and retraction so destroyed that the mother's life is endangered by the probability of post-partum haemorrhage, if not by conditions arising antecedent to the birth of the child.

To draw conclusions from one case can only be justified by the rarity of the condition, and by the hope that others will compare such conclusions with their own personal experiences. The condition has been attributed to toxaemia, trauma, emotional disturbances, and endometritis, and is commoner in multiparae. This case throws no light on the subject, but the date and hour of its onset are significant-10 p.m., December 31st.

Diagnosis offer's no difficulty. The sudden onset of abdominal pain in a woman advanced in pregnancy, with marked collapse and with no "show," should suggest the condition. The tense hard swelling of the uterus, particularly in its upper segment, with inability to hear the foetal heart sounds or uterine souffle, are sufficient to confirm the diagnosis.

The sequence of events following haemorrhage into the decidua basalis must depend on the amount of blood poured out of the ruptured ressels and on the increased intrauterine pressure resulting. As long as the haemorrhage remains concealed between the placenta and membrane on the one hand and the uterine wall on the other the increased pressure acting through the liquor amnii tends to dilate the os to some extent, at the same time ballooning the uterus, and disseminating the fluid blood between the bundles of uterine muscle. This sequence of erents would contribute to a lowering of the intrauterine pressure if the haemorrhage ceased, but in reality it encourages further bleeding, with increased distension of the uterus and greater dissociation of muscle bundles until the intraabdominal pressure is raised to or above that in the uterus; then the blood sinuses at the placenta site are subjected to compression which closes them and permits thrombosis to occur. It might be argued that such a state will occur earlier in a primipara or in a multipara with well dereloped and not overstretched abdominal muscles. Conrersely, lax abdominal muscles will allow so great uterine distension that the peritoneum cracks and the dissociated muscle bundles are converted into actual ruptures permitting blood to escape into the abdominal cavity. Such cases have been described by Shannon and others. If this case can be taken as a criterion of what occurs in others, then the distension is limited to the uterine body, while the lower segment is merely stretched longitudinally and is otherwise not traumatized by the disseminating blood.

If the diagnosis could be made early, the ideal treatment would be to convert the concealed into an external haemorrhage, but as the first symptoms of concealed accidental haemorrhage arise from shock due to the rapid distension of the uterus, most cases are seen too late for this to be done without considerable risk. The firm application of an abdominal binder may assist in raising the intraabdominal pressure and minimizing the haemorrhage.

To empty the uterus per vias naturales endangers the patient's life from post-partum haemorrhage, whereas if it is emptied through an abdominal hysterotomy opening the post-partum haemorrhage can be controlled by direct compression or by hysterectomy if other measures fail. If Caesarean section is performed the lower uterine segment operation appears to be more desirable than the classical method. It is the part of the uterus underlying a mid-line incision below the umbilicus; it is stretched longitudinally and there is ample room without the necessity of reflecting the bladder downwards; it is not traumatized, at least to the same extent, as is the body; and if the uterus should become infected it is shut off from the peritoneal cavity.

The collapsed and ivory-white condition of the intestines is difficult to explain, especially as no reaction appeared during the twenty to twenty-five minutes the abdomen was open. The greatly increased intra-abdominal pressure, with irritation of the splanchnic nerves, may have had some influence in producing this state, or it may be a phenonenon of shock as described by some writers on that subject.

\section{A PROTEIN POISON THEORY:}

\section{Its Application to the Treatment of Headache and Especially Migrifine.}

BY

\section{R. CURTIS-BROWN, M.D.,}

MENTONE, FRANCE.

ALI nitrogenous food, animal or vegetable, contains a potential poison. Whether untoward effects are produced or not depends entirely upon the metabolic efficiency of the indiridual in respect of the particular protein concerned. If the metabolic capacity of an individual is insufficient to deal with this poison, without undue reaction, manifestations are produced. These manifestations may occur in any tissue of the body, and are the symptoms of disease.

In chronic disease the reactions are so slight that they are not erident until the gradual replacement of the destroyed tissue cells by connective tissue interferes with the function of an organ. This explains the slow development of arteriosclerosis, nephritis, and other diseases of like nature. On the other hand, the manifestations may be sudden and serere, as in cyclic vomiting and migraine. Most acute manifestations show a certain periodicity, indicating a cumulatire action of the poison. Other acute affections appear immediately after the ingestion of the poison, as 